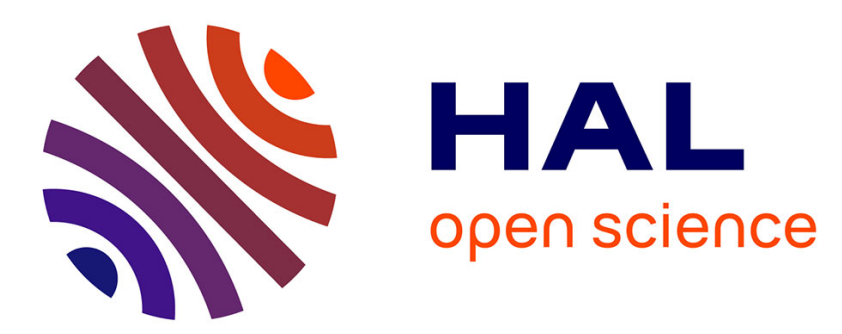

\title{
Hybrid Model: Permeance Network and 3-D Finite Element for Modeling Claw-Pole Synchronous Machines
}

Geoffrey Devornique, Julien Fontchastagner, Denis Netter, Noureddine

Takorabet

\section{- To cite this version:}

Geoffrey Devornique, Julien Fontchastagner, Denis Netter, Noureddine Takorabet. Hybrid Model: Permeance Network and 3-D Finite Element for Modeling Claw-Pole Synchronous Machines. IEEE Transactions on Magnetics, 2017, 53 (6), pp.7206704. 10.1109/TMAG.2017.2657890 . hal-01631082

\section{HAL Id: hal-01631082 \\ https://hal.univ-lorraine.fr/hal-01631082}

Submitted on 8 Nov 2017

HAL is a multi-disciplinary open access archive for the deposit and dissemination of scientific research documents, whether they are published or not. The documents may come from teaching and research institutions in France or abroad, or from public or private research centers.
L'archive ouverte pluridisciplinaire HAL, est destinée au dépôt et à la diffusion de documents scientifiques de niveau recherche, publiés ou non, émanant des établissements d'enseignement et de recherche français ou étrangers, des laboratoires publics ou privés. 


\title{
Hybrid Model: Permeance Network and 3D Finite Element for Modeling Claw-Pole Synchronous Machines
}

\author{
Geoffrey Devornique, Julien Fontchastagner, Denis Netter, and Noureddine Takorabet \\ Université de Lorraine, GREEN, Vandoeuvre-lès-Nancy, F-54500, France.
}

\begin{abstract}
Designing a claw-pole synchronous machine implies solving many 3D nonlinear magnetostatic problems which makes the computation (CPU) time very long. In our model, the mesh is refined to reach the desired level of precision on global quantities such as torque. Since the airgap is very thin (around $0.3 \mathrm{~mm}$ for a $100 \mathrm{~mm}$ diameter) and a Newton Raphson algorithm requires several iterations to converge, CPU time may be too high. Nowadays, many researches are ongoing to reduce the CPU time, while preserving an acceptable accuracy. One of the most efficient methods is permeance networks but this method is not suitable for complex geometries. Our main contribution is to use a permeance network in the areas where flux lines are easy to guess and to solve a 3D FEM problem in complex geometry areas of the magnetic device: the claw poles and the air gap for example. Moreover, current sources belong to the permeance network model, so that there are no current sources in the 3D FEM problem. Then, a 3D scalar magnetic potential formulation can be used easily. The two classical magnetostatic formulations (magnetic scalar potential formulation $\left(U_{m}-h_{s}\right)$ and vector potential formulation $(a-j)$ ) are presented in this paper. Then, the hybridization of 3D FEM formulation and the permeance network, is presented. Numerical results are compared with experimental measurements and a good agreement is obtained while reducing the CPU time.
\end{abstract}

Index Terms-Claw-pole synchronous machine, 3D FEM, permeance networks, hybrid models.

\section{INTRODUCTION}

$\mathrm{T}$ HE claw-pole synchronous machine (CP-SM), shown in Fig. 1 is widely used in the automotive industry thanks to its low-cost price and robustness compared to other electrical machines. The complex geometry of claws in the rotor makes it difficult to model with accuracy the CP-SM, whatever the method (numerical or analytical). It cannot be considered as a 2D geometry and it has to be kept unaltered. Besides, the 3D effects due to the claw poles cause local high magnetic saturation, mainly in the rotor claws and stator teeth. These two characteristics lead to solve a difficult nonlinear 3D magnetostatic problem. Reducing the CPU time is a real challenge.

There are many strategies to obtain the optimum balance between CPU time and accuracy. The main idea is to mix an analytical method based on a permeance network with a numerical one based on 3D finite element (FE) method. On one hand, the 3D FEM takes into account the real motor shape without geometric approximations, and gives accurate results. But computation time is high. On the other hand, the analytical method uses less computing resources, at the expense of accuracy [1], [2].

Our main contribution is to take advantage of these two complementary approaches for a 3D problem. To do this, a coupling between permeance networks and FE is carried out with free finite element softwares (the mesh tool Gmsh [3] and the solver GetDP [4]) but it could be implemented in any other computation tool allowing a coupling of electric circuits with nonlinear resistances and FEM.

The machine is cut into two areas. A permeance network is built from the ferromagnetic areas where the field lines are twodimensional (the upper stator armature and the central part of the rotor). The air gap and the areas where flux leaks are important (claws and slots) will be the area modeled FEM.
In section II, the choice of magnetostatic formulation is presented as well as the hybridization of 3D FEM with permeance network. Then, in section III, the hybrid model and experimental results are compared.

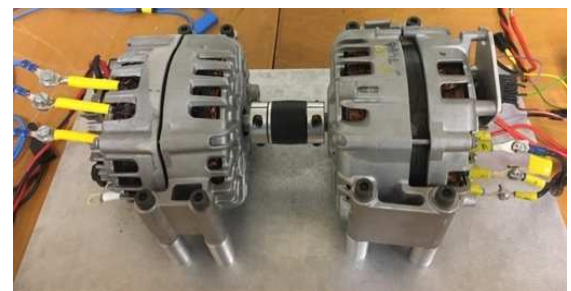

Fig. 1. Experimental test bench with two synchronous claw-pole machine

\section{Magnetic Modelling}

To compare the performances of the different approaches, we use global quantities: the no-load electromotive force (emf) and the torque $(\Gamma)$. To obtain these different global quantities, two types of models are possible: a casual FE model on the whole geometry and the proposed hybrid model.

\section{A. Classical FE 3D models}

Both conventional models rely on the use of magnetic potentials: the scalar potential $\left(U_{m}\right)$ or the vector potential $(\boldsymbol{a})$. These two potentials bring us either to a b-conforming or to a h-conforming formulation, which provide two variational formulations related to (1) and (2).

$\left\langle v \operatorname{curl}(\boldsymbol{a}), \operatorname{curl}\left(\boldsymbol{a}^{\prime}\right)\right\rangle_{\Omega}=\left\langle\boldsymbol{j}, \boldsymbol{a}^{\prime}\right\rangle_{\Omega_{S}}+\left\langle v \boldsymbol{b}_{r}, \operatorname{curl}\left(\boldsymbol{a}^{\prime}\right)\right\rangle_{\Omega_{M}}$

with $j$ the current density, $v$ the reluctivity and $\boldsymbol{b}_{\boldsymbol{r}}$ the remanence, $\Omega$ the studied domain, $\Omega_{\mathrm{s}}$ the source domain and $\Omega_{M}$ the permanent magnet domain. 
$\left\langle\mu\left(\boldsymbol{h}_{\boldsymbol{c}}+\boldsymbol{h}_{\boldsymbol{s}}\right), \boldsymbol{g r a d}\left(U_{m}^{\prime}\right)\right\rangle_{\Omega}$

$$
-\left\langle\mu \operatorname{grad}\left(U_{m}\right), \operatorname{grad}\left(U_{m}^{\prime}\right)\right\rangle_{\Omega}=0
$$

with $\boldsymbol{h}_{s}$ the source field $\left(\boldsymbol{h}_{s}=\boldsymbol{c u r l}(\boldsymbol{j})\right), \boldsymbol{h}_{\boldsymbol{c}}$ is the coercive field, $\mu$ the permeability.

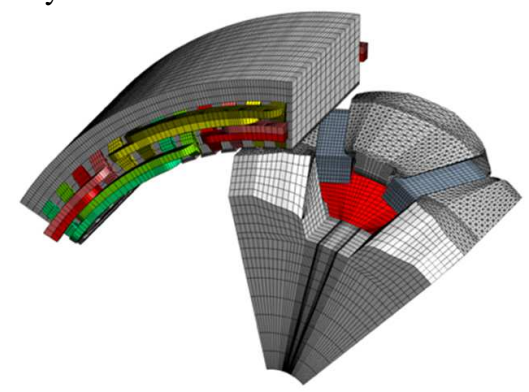

Fig. 2. The 3D FE model

The studied machine, shown in Fig. 2, is purely threedimensional. In the case of $(\boldsymbol{a}-\boldsymbol{j})$ formulation, a spanning tree gauge [5] is implemented to ensure the magnetic vector potential uniqueness. The current density is imposed by analytic formulas for the rotor coil and computed by a current-flow preresolution for the stator coils. However, for $\left(U_{m}-\boldsymbol{h}_{s}\right)$ formulation, we must replace the current sources by an equivalent source field. Among the various methods to evaluate this source field, we choose an indirect method proposed by Dular_et al. in [6]. It can be applied to any shape of inductor while reducing CPU time.

The scalar formulation has fewer unknowns than the vector formulation. However, computing the source field in a periodic geometry with complex coils is difficult and causes cumulative errors, because of an additional step. We note that the calculation of the emf by the flux derivative is easier with the $(\boldsymbol{a}-\boldsymbol{j})$ formulation than with the $\left(U_{m}-\boldsymbol{h}_{\boldsymbol{s}}\right)$. In return, the $(\boldsymbol{a}-\boldsymbol{j})$ formulation needs more computing resources due to the size of the system.

To show objectively that the hybrid-method presented later is efficient, we did our best to speed up the convergence of $(\boldsymbol{a}-$ $\boldsymbol{j})$ and $\left(U_{m}-\boldsymbol{h}_{s}\right)$ formulations. Therefore, we use two different solvers with the same convergence criterion. A NewtonRaphson method with a convergence criterion of $\|\boldsymbol{b} . \boldsymbol{h}\|$ in the air-gap with a relative accuracy of $10^{-3}$ is selected for the $(\boldsymbol{a}-$ $j$ ) formulation. According to the literature, the Newton-Raphson method is not the most suitable for $\left(U_{m}-\boldsymbol{h}_{s}\right)$ formulation [8]. For the $\left(U_{m}-\boldsymbol{h}_{s}\right)$ formulation, a fixed point method with a linear pre-initialization is implemented with the same convergence criterion. The choice of solvers and convergence criterion results from our previous works to reduce $\mathrm{CPU}$ time in the case of a nonlinear magnetostatic problem [7].

\section{B. Hybrid formulation}

The hybrid formulation is based on additional assumptions. It can be understood as the mix of FEM and permeance network. The 3D FEM unknowns are either $\boldsymbol{a}$ or $U_{m}$ depending on the chosen formulation. The permeance network unknowns are the flux $(\varphi)$ or the magnetomotive force $\left(V_{p}\right)$. As usual, we assume that the flux density $\boldsymbol{b}$ is oriented along the length such as $L_{e q}$ and uniform throughout a flux tube, so that we can write:

$$
\left\{\begin{array}{l}
\varphi=\iint \boldsymbol{b} \cdot d \boldsymbol{S}=B S_{e q} \\
V_{p}=\int \boldsymbol{h} \cdot d \boldsymbol{l}=H L_{e q}
\end{array}\right.
$$

where $S_{e q}$ and $L_{e q}$ represent respectively, the equivalent surface (supposed constant) and the average length of the flux tube.

Considering the magnetic constitutive law of the ferromagnetic material, permeance $(P)$ or reluctance $(R)$ can be written as:

$$
\left\{\begin{array}{c}
R(\varphi)=\frac{L_{e q}}{\mu\left(\frac{\varphi}{S_{e q}}\right) S_{e q}} \text { such as } V_{p}=R(\varphi) \varphi \\
P\left(V_{p}\right)=\mu\left(\frac{V_{p}}{L_{e q}}\right) \frac{S_{e q}}{L_{e q}} \text { such as } \varphi=P\left(V_{p}\right) V_{p}
\end{array}\right.
$$

$R$ and $P$ depend on $B$ and $H$ through the magnetic permeability and the permeance network is made of nonlinear components. This hybrid method can be implemented in any software under the condition that it supports nonlinear electrical circuits, especially resistances.

In addition to reducing the unknowns number, permeance network method enables to avoid the calculation of the source field in the case of a $\left(U_{m}-\boldsymbol{h}_{s}\right)$ formulation. We propose to remove the current density distribution in FEM domain and to replace it by an equivalent ampere-turns (NI) source in the permeance network domain. Since there are no more currents in the FEM domain, we end up with a currentless problem for which a $U_{m}$ formulation is the most suitable. The scalar potential exists everywhere, even in the slots, and the question no longer arises for the calculation of the source field. Besides, with this approach, fluxes are directly available after resolution. We will see in section III that it does not impact too much the flux density distribution in the air gap and global quantities (emf and torque). The magnetic field in the FEM domain is no longer due to a volumic current density distribution but to a flux source imposed at the boundary of the FEM domain.

Taking as an example the CP-SM, the flux density is threedimensional in the stator teeth, the link between FE method and permeances will be located at the top of stator teeth. Thereby the field sources will be injected at the top of the slots as shown in Fig. 3.
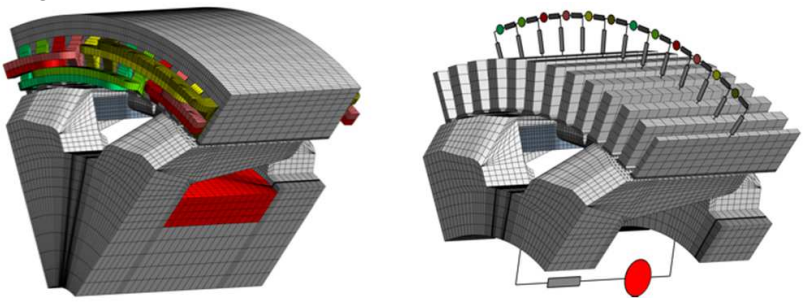

Fig. 3. Full 3D FE model and corresponding hybrid model

The middle part (FE domain) is only made of ferromagnetic and magnets. The rotor yoke and the excitation coil are replaced by the permeance circuit at the inner radius (bottom) whereas the stator yoke and coils are replaced by the permeance network on the outer radius (top). Ampere-turns (NI) sources are 
represented by circles, permeances by rectangles.

The hybrid model we propose is made of three equations, for which unknowns are scalar potential $\left(U_{m}\right)$ and magnetomotive forces $\left(V_{p}\right)$. The first one is the variational $U_{m}$ formulation in the FE domain. Compared to the formulation (2), it includes an additional term taking into account the fluxes on its boundary due to the reluctance network. The second equation is a set of nonlinear algebraic equations which link fluxes $\varphi$ and potentials $V_{p}$ in the reluctance network. It can be written as the variational equation (6) (see [9] and [10] for details). The sources of magnetomotive forces are implemented as a constraint in the corresponding function space. That is why NI sources do not appear in the variational formulation. The third relation provides the coupling between the last two formulations: fluxes on the surfaces $\mathrm{d} S_{c}$ are expressed from both FE formulation in $\Omega_{F E}$ and permeance network formulation in $\Omega_{\text {circ }}$. The flux of each branch of the permeance network is injected into the ferromagnetic surface connected to this permeance, so that the flux density $\boldsymbol{b}$ is supposed to be purely normal to the connection surface. This is a simplifying assumption.

$$
\begin{gathered}
\left\langle\mu \boldsymbol{h}_{\boldsymbol{c}}, \boldsymbol{g r a d}\left(U_{m}^{\prime}\right)\right\rangle_{\Omega_{F E}}-\left\langle\mu \operatorname{grad}\left(U_{m}\right), \boldsymbol{g r a d}\left(U_{m}^{\prime}\right)\right\rangle_{\Omega_{F E}} \\
+\left\langle\boldsymbol{n} \cdot \boldsymbol{b}, U_{m}^{\prime}\right\rangle_{\mathrm{dS}_{C}}=0 \\
\left\{\begin{array}{c}
\left\langle[\varphi],\left[V_{p}^{\prime}\right]\right\rangle_{\Omega_{c i r c}}-\left\langle[P]\left[V_{p}\right],\left[V_{p}^{\prime}\right]\right\rangle_{\Omega_{c i r c}}=0 \\
\varphi_{i}=\iint_{d S_{c_{i}}} \boldsymbol{n} \cdot \boldsymbol{b} d S \Rightarrow[\varphi]=\left\langle\boldsymbol{n} \cdot \boldsymbol{b}, U_{m}^{\prime}\right\rangle_{\mathrm{dS}_{C}}
\end{array}\right.
\end{gathered}
$$

The flexibility of Gmsh - GetDP allows us to implement the variational formulation governing the hybrid model.

\section{Implementation of the $\left(U_{m}-V_{p}\right)$ hybrid model}

The weak formulation (5) uses linear scalar nodal elements. This basis functions are based on a structured mesh as shown in Fig. 3 and 4. Mesh in the FE of the hybrid model $\left(\Omega_{\mathrm{FE}}\right)$ is the same as in the two other full FE models. Periodic conditions are used to simplify the problem. A rolling band made of three elements is used in the airgap which length is $0.3 \mathrm{~mm}$. The nonlinear hybrid formulation is solved by using a fixed point method in the whole domain (FE and permeance network) via a strong coupling.

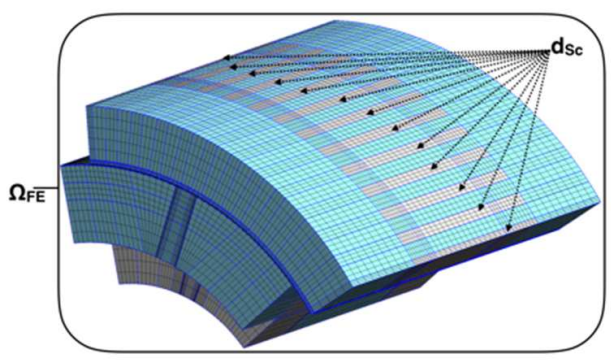

Fig. 4. Gmsh-GetDP implementation of the hybrid model

The first step of validation consists in comparing the two ( $\boldsymbol{a}$ $-\boldsymbol{j})$ and $\left(U_{m}-\boldsymbol{h}_{s}\right)$ formulations with the proposed hybrid formulation. Fig. 5 and 6 show the flux density distribution in the ferromagnetic parts of FE domain for a full-load operation. Maximum values are quite the same but so are b-distributions. The next section deals with a deeper comparison.
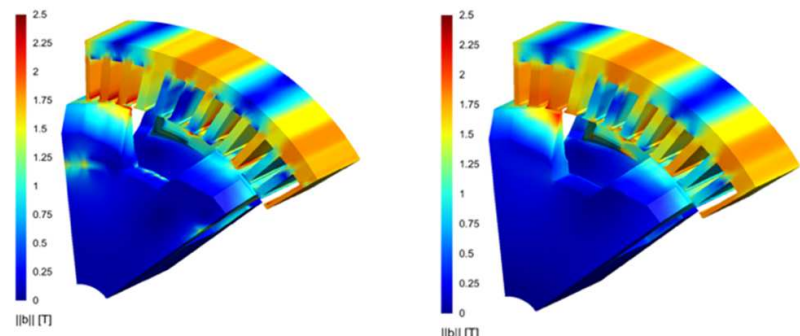

Fig. 5. $\|\mathbf{b}\|$ for a load operation for $(\mathbf{a}-\mathbf{j})$ and $\left(U_{m}-\mathbf{h}_{\mathrm{s}}\right)$ formulations

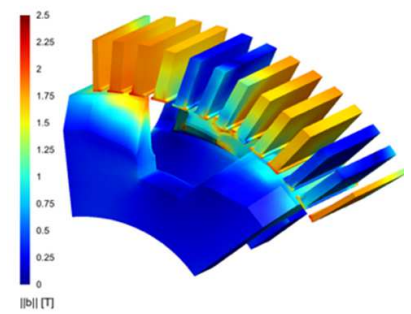

Fig. 6. $\|\mathrm{b}\|$ for a load operation for the $\left(U_{m}-V_{p}\right)$ hybrid model

\section{RESULTS}

In this section, we will discuss about a no-load and a fullload operations. We can provide experimental measurements for the no-load operation and we will compare the emf given by two numerical formulations and experimental results. For the full-load operation, as our test bench does not have any torque sensor yet, three numerical models are compared.

The emf are calculated by derivating the magnetic fluxes. In the hybrid model, the fluxes in the network branches are directly available after the resolution and it only has to sum them to obtain the total flux $(\phi)$ through the coil $\operatorname{coil}_{i}$. In the case of FE periodic model with $(\boldsymbol{a}-\boldsymbol{j})$ formulation, (7) is implemented.

$$
\phi_{i}=\frac{1}{I} \iiint_{\text {coil }_{i}} \boldsymbol{a} \cdot \boldsymbol{j} d V \text { and } E_{i}=-\Omega \frac{\mathrm{d} \phi_{i}}{\mathrm{~d} \theta}
$$

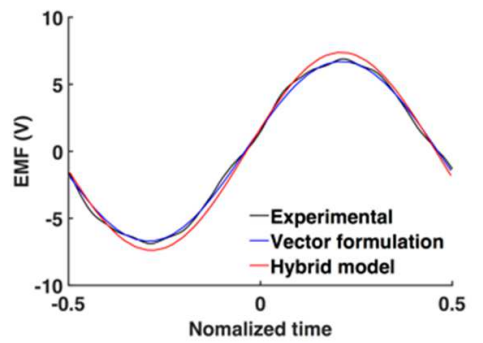

Fig. 7. EMF over one period

Fig. 7 shows the no-load emf waveforms whereas Fig. 8 plots the rms value of emf versus excitation current $\left(I_{f}\right)$, for a constant rotor speed. For $I_{f}=0$, emf is non zero because the machine includes both wounded inductor and magnets. As expected, the 
$(\boldsymbol{a}-\boldsymbol{j})$ formulation gives emfs very close to measured ones because this model uses the least simplifying assumptions. The relative difference for the no-load emf is less than $8 \%$ in the worst case compared to both experimental and full 3D results.

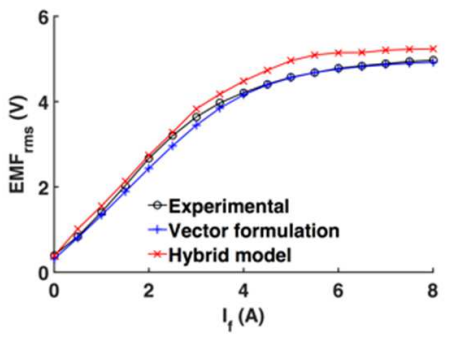

Fig. 8. EMF versus $I_{f}$

In the case of full-load operation, the torque is calculated by the volume integral of the Maxwell stress tensor in the movingband using (8). The volume integral enables to average surface integrals and it naturally smooths the curve Torque waveform.

$\Gamma=\frac{p}{e_{c}} \iiint_{\Omega_{C}} \frac{\left(B_{x} B_{y} \cdot\left(x^{2}+y^{2}\right)+\left(B_{y}^{2}-B_{x}^{2}\right) x y\right)}{\mu_{0} \sqrt{x^{2}+y^{2}}} d x d y d z$

As shown in Fig. 9, the hybrid model underestimates the average torque (under $10 \%$ of difference) due to the different assumptions of permeance network model. This type of models allows us to take into account the real 3D effect inside the claw. In a future work, this model will be used for the optimization of claw shape for a given stator geometry.

TABLE I

TORQUE SiMULATIONS

\begin{tabular}{lccc}
\hline \hline models & $(\boldsymbol{a}-\boldsymbol{j})$ & $\left(U_{m}-\boldsymbol{h}_{s}\right)$ & $\left(U_{m}-V_{p}\right)$ \\
\hline$\Gamma_{\mathrm{m}}(\mathrm{N} . \mathrm{m})$ & 21.76 & 21.68 & 20.31 \\
CPU time for a & 356 & 1423 & 165 \\
given rotor angle $(\mathrm{s})$ & & & \\
DOFs number & 450650 & 226318 & 134583 \\
Iteration number & 11 & 23 & 12 \\
\hline \hline
\end{tabular}

From Table I, we can conclude that the $\left(U_{m}-V_{p}\right)$ hybrid model, even if it is based on strong assumptions, gives a quite good approximate value of the average torque. Moreover, CPU time is divided by 2 . Besides, DOFs number is at least divided by 4 . The gain with the $\left(U_{m}-\boldsymbol{h}_{s}\right)$ formulation is much higher: CPU time is divided by 8.6 .

CPU time is more or less the same for no-load emf and full load torque. Our hybrid model will really improve the convergence speed of optimization processes, without damaging the accuracy. Our work began with a simpler device (electromagnet) and we drew the same conclusions. This example proves the robustness of the $\left(U_{m}-V_{p}\right)$ formulation Even in the case of a complex geometry.

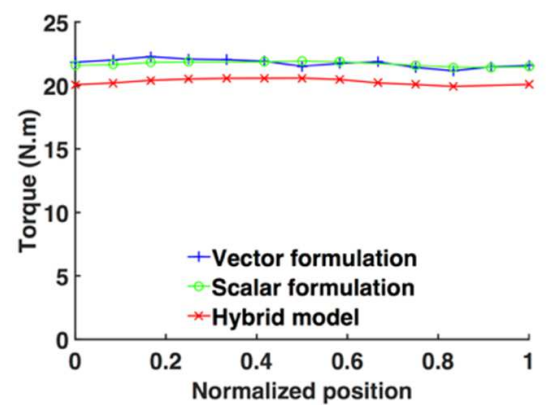

Fig. 9. Torque waveform over slot pitch

\section{CONCLUSION}

The aim of this work is to speed up optimization processing. An original model based on hybridization of 3D FE and permeance network is applied to a claw-pole machine. The $\left(U_{m}\right.$ $-V_{p}$ ) formulation has been chosen for several reasons. First, the scalar potential $U_{m}$ and $V_{p}$ are easier to link together at the common boundary because $\operatorname{grad}\left(U_{m}\right)$ is similar to a potential difference $V_{p}$. Moreover, current sources are transferred in the permeance network which avoids calculating any source field for the scalar potential formulation. It provides accurate results and CPU time is reduced, which makes it suitable for pre-design and/or optimization purposes. We have ongoing studies concerning other hybrid formulations, such as $(\boldsymbol{a}-\boldsymbol{\varphi})$.

\section{REFERENCES}

[1] V. Ostovic, J. M. Miller, V. K. Garg, R. D. Schultz and S. H. Swales, A Magnetic-Equivalent-Circuit Based Performance Computation of a Lundell Alternator, in IEEE Transactions On Industry Applications, vol. 35 , no. 4, pp. 825-830, 1999.

[2] L. Albert, C. Chillet, A. Jarosz and F. Wurtz, Analytic Modeling of Automotive Claw-Pole Alternator for Design and Constrained Optimization, in Proc. $10^{\text {th }}$ Eur. Conf. Power Electronics and Applications, Toulouse, France, Sept. 24, 2003.

[3] C. Geuzaine and J.-F. Remacle, Gmsh: a three-dimensional finite element mesh generator with built-in pre- and post-processing facilities, International Journal for Numerical Methods in Engineering, vol. 79, no. 11, pp. 1309-1331, 2009.

[4] P. Dular, C. Geuzaine, F. Henrotte and W. Legros, A general environment for the treatment of discrete problems and its application to the finite element method, IEEE Transactions on Magnetics, vol. 34, no. 5, pp. 3395-3398, 1998.

[5] P. Dular, A. Nicolet, A. Genon, and W. Legros., A discrete sequence associated with mixed finite elements and its gauge condition for vector potentials, IEEE Transactions on Magnetics, vol. 31, no. 3, pp. 13561359, 1995.

[6] P. Dular, P. Kuo-Peng, C. Geuzaine, N. Sadowski, and J.P.A. Bastos, Dual magnetodynamic formulations and their source fields associated with massive and stranded inductors, IEEE Transactions on Magnetics, vol. 36, no. 4, pp. 1293-1299, 2000.

[7] G. Devornique, J. Fontchastagner, N. Takorabet and D. Netter, Fast Computation of Nonlinear 3D FE Analysis for Electrical Machines, in Proc. $10^{\text {th }}$ Int. Sym. On Electric and Magnetic fields EMF, Lyon, France, April, 2016.

[8] C. Neagoe and F. Ossart, Analysis of convergence in nonlinear magnetostatic finite elements problems, IEEE Transactions on Magnetics, vol. 30, no. 5, pp. 2865-2868, 1994.

[9] P. Dular and W. Legros, Coupling of local and global quantities in various finite element formulations its application to electrostatics, magnetostatics and magnetodynamics, IEEE Transactions on Magnetics, vol. 34, no. 5, pp. 3078-3081, 1998.

[10] P. Dular, J. Gyselinck, T. Henneron and F. Piriou, Dual finite element formulations for lumped reluctances coupling, IEEE Transactions on Magnetics, vol. 41, no. 5, pp. 1396-1399, 2005. 